\title{
Modelling acoustic scattering, sound speed and attenuation in gassy soft marine sediments
}

A. Mantouka, H. Dogan, P. R. White and T. G. Leighton ${ }^{a}$

Institute of Sound and Vibration Research, Faculty of Engineering and the Environment, University of Southampton, Highfield, Southampton, SO17 1BJ

Running title: Acoustics in gassy marine sediments

\begin{abstract}
A model for nonlinear gas bubble pulsation in marine sediments is presented. This model is then linearized to determine the resonance frequency and the damping terms for linear radial oscillations. The linear model is then used to predict the effects that such bubble pulsations will have on the sound speed and attenuation of acoustic waves propagating in gassy marine sediment. The results are compared for monodisperse populations against the predictions of a model of Anderson and Hampton and, furthermore, the additional abilities of the new model are discussed. These features include the removal of the sign ambiguities in the expressions, the straightforward implementation for acoustic propagation through polydisperse bubble populations, the capability to estimate bubble size distributions through a full acoustic inversion, and the capability to predict nonlinear effects.
\end{abstract}

PACS: 43.30.Ma, 43.25.Yw, 43.30.Pc, 43.40.Ph

\footnotetext{
${ }^{\mathrm{a}}$ Corresponding author: T. G. Leighton FREng FRS, T.G.Leighton@soton.ac.uk
} 


\section{INTRODUCTION}

Characterisation of the amount of gas in marine sediment is important for several reasons. Firstly, for understanding the stability of the sediment with respect to both natural disturbances, such as undersea landslip, and manmade processes, such as piling for civil engineering projects [1-3]. Secondly, for assessment of the gas reserves for fuel and climate risk [4-7]. Such quantification of marine gas reserves should also take into account that which could potentially dissociate from hydrate. Thirdly, for monitoring leakages into sediment from buried manmade gas bodies, including buried gas pipelines and carbon capture and storage facilities [4, 8-10]. Fourthly, the resonance of gas bubbles in sediment can profoundly perturb the propagation and scattering of acoustic waves. Accurate models of these effects are needed to predict the performance of the systems that might, in situ, involve propagating waves through gassy sediments (e.g. for sending data to and from buried transponders, for sub-bottom surveying [11] and for in situ calibration [12]). Accurate models are also needed if the measured attenuation and sound speed are to be inverted to estimate the gas bubble population present in the sediment. The current models for bubble dynamics in sediments are insufficient for inverting measured acoustical propagation and scattering in order to estimate the bubble populations present in the seabed, unless very specific conditions are assumed (e.g. all bubbles are much smaller than the bubble size that resonates with the sound field [11]). Furthermore, since the attenuation can be high, the source levels used (to provide an adequate signal-to-noise ratio at the receiver) might generate nonlinear bubble oscillations for part of the propagation path, and indeed some methods for measuring or exploiting sub-bottom bubbles might rely on such nonlinearities [13-18]. Consequently, the inclusion of bubble nonlinearities into such models could, at times, be important [19, 20].

Acoustic propagation in marine sediments has been extensively investigated. The forward models mainly falling into the following categories: A large body of literature assumed the existence of one compressional and one shear wave, and explored ways to model the frequency dependence of attenuation, $\alpha(\mathrm{dB} / \mathrm{m})$, with the following empirical relation [21]:

$$
\alpha=\Gamma f^{p},
$$


where $p$ is the attenuation exponent, $f$ is the frequency and $\Gamma$ is a constant. There is a broad range of reported values covering a variety of marine sediments [22-33] although the reported values of $p$ are not consistent between experiments, most probably because of the different sediment types and frequency ranges considered. The value of $p$ is frequently reported as representing a linear model of attenuation (e.g. Wood and Weston [34], and Bowles [35]). However, there are also a number of studies that report attenuation exponents which exhibit non-linear attenuation relationships (e.g. Stoll [36], Brunson and Johnson [37], and Turgut and Yamamoto [32]).

In water-saturated sediment, Biot [38, 39] showed the existence of two compressional waves and one shear wave; this has been firmly validated through experimental work [36, 40, 41]. Although Biot's work initially suggested negligible dispersion of compressional waves, later studies have reported significant dispersion in a wide range of excitation frequencies, i.e. 1-100 $\mathrm{kHz}$ [42] or $10 \mathrm{kHz}-1 \mathrm{MHz}$ [43]. The majority of the studies which aimed to model the inclusion of gas bubbles into porous sediment have assumed that the bubbles are contained only in the pore fluid spaces and they do not affect the sediment structure [44-46]. This assumption implies that the contact between the gas bubble and the sediment mineral grains are not taken into account; hence, those models are not applicable to the 'sediment-replacing' type of bubble in the classification of Anderson et al. [47]. To our knowledge, the work of Kargl et al. [48] is the only publication that takes into account the effects of sediment rigidity on the pulsations of bubbles and the presence of two compressional waves, and investigates the double resonance behaviour of bubbles under the effect of two compressional waves. However, their model leaves out the formulation of damping, sound speed and attenuation relevant to bubble pulsations and does not provide a route for acoustic inversion. The notable contribution of Boyle and Chotiros [49, 50] also formulated the propagation of two compressional waves in sediment containing gas bubbles, although the viscoelastic damping was attributed to the pore water viscosity only, which may result in underestimated attenuation caused by bubbles.

Anderson and Hampton [51, 52] presented the model that is most frequently used for predictions of acoustic scattering, sound speed and attenuation in gas-bearing marine sediments. The applicability of this model is limited to linear gas bubble pulsations, and leaves ambiguities for the inverse problem because of the plus/minus sign entering the expression of 
the complex sound speed (equation 49 of [51]). This sign ambiguity may result in the presence of both positive and negative bubble counts when inverting broadband acoustic data. For instance, Best et al. [53] measured the attenuation spectra between 600 and $3000 \mathrm{~Hz}$ and identified five peaks which were attributed to bubble resonances. The attenuations for these single bubble sizes were computed theoretically (their Figure 9a, 9b) in order to obtain the bubble number density, which were then weighted to estimate a continuous bubble size distribution (their Figure 10). However, the weightings with best fit to the measured attenuation gave a poor fit to the sound speed data (their Figure 9c, 9d). The method outlined in this paper, on the other hand, is suitable for a full acoustic inversion which satisfies both sound speed and attenuation data simultaneously [54].

Lyons et al. [55] have also performed predictions of bubble populations in gassy sediment using the linear scattering cross section expressions (equations 54-56) of Anderson and Hampton (equations that are not self-consistent [56, 57]). The model presented in Section I is suitable for implementation with short high amplitude pulses (as required for range resolution [58]), and for nonlinear inversions (e.g. when the high amplitudes required to overcome sediment attenuation produce harmonics, ultraharmonics and subharmonics in the bubble wall displacements [54] or produce nonlinear mixing of two insonification frequencies [59-61], a technique which is receiving increasing interest in sediment studies [16, 17, 62, 63]).

In this work, it is assumed that the gas-free host medium supports one compressional and one shear wave. Furthermore, the dispersion of the first compressional wave and the dissipation due to internal friction are neglected. Whilst more comprehensive studies should address mismatches between such assumptions and observations, that is beyond the scope of this article, which restricts itself to the regime of assumptions about dispersion that were used in the model of Anderson and Hampton, against which the predictions of the new model are compared. Incorporation of the second compressional wave and the other losses into the model requires a more comprehensive treatise, i.e. by expanding the formulation of Kargl et al. [48] to a nonlinear setting. However, this is not a straightforward task since the Biot theory itself is inherently linear. The effective bulk and shear moduli of the host medium, on the other hand, is calculated in this work using a comprehensive model based on Berryman's self-consistent approximation $[64,65]$. 


\section{BUBBLE DYNAMICS}

Leighton [20] presented a nonlinear time-dependent model for bubble dynamics in viscoelastic medium, i.e. marine sediments. It incorporated the shear properties of the host medium, and that paper identified that the next stage in developing that formulation would be to replace the assumption of an incompressible host medium with one that includes acoustic radiation losses. This is the objective of the current paper, which combines the general form of the KellerMiksis equation with the linear Voigt viscoelastic model.

Consider the radial motion of a spherical gas bubble of radius $R(t)$ which is fixed in space and oscillates about some equilibrium radius $R_{0}$ with bubble wall velocity $\dot{R}(t)$. Assuming the flow is irrotational (implying that the porosity is a homogenous property of the host medium) yields the following equation for radial bubble dynamics which accounts for the compressibility of the surrounding medium to first order [66]:

$$
\begin{aligned}
\rho_{\mathrm{s}}\left(1-\frac{\dot{R}}{c_{\mathrm{p}}}\right) R \ddot{R} & +\frac{3}{2} \rho_{\mathrm{s}}\left(1-\frac{\dot{R}}{3 c_{\mathrm{p}}}\right) \dot{R}^{2} \\
& =\left(1+\frac{\dot{R}}{c_{\mathrm{p}}}+\frac{R}{c_{\mathrm{p}}} \frac{d}{d t}\right)\left(p_{\mathrm{g}}-\frac{2 \sigma}{R}-p_{0}+P_{\mathrm{A}} g(t)-\frac{4 \eta_{\mathrm{s}} \dot{R}}{R}-\frac{4 G_{\mathrm{s}}}{3 R^{3}}\left(R^{3}-R_{0}^{3}\right)\right)
\end{aligned}
$$

Equation (2) was first proposed by Yang and Church [66] in order to model the acoustic propagation in viscoelastic tissues; it is also applicable to marine sediments provided that the appropriate viscoelastic constitutive relation is employed. It inherits the acoustic radiation losses; several routes for the incorporation of such losses into a Rayleigh-Plesset type equation were outlined in Ref. [20].

In (2), $G_{\mathrm{s}}$ is the shear modulus, $\eta_{\mathrm{s}}$ denotes the shear viscosity, $p_{0}$ is the static pressure outside the bubble, $p_{\mathrm{g}}$, is the pressure within the bubble and $\sigma$ denotes the surface tension at the bubble wall. The equilibrium gas pressure $p_{\mathrm{g}_{0}}$ can be expressed in terms of $p_{0}$, the equilibrium Laplace pressure $\left(p_{\sigma, 0}=2 \sigma / R_{0}\right)$ and vapour pressure $p_{\mathrm{v}}$ as: 


$$
p_{\mathrm{g}_{0}}=p_{0}+\frac{2 \sigma}{R_{0}}-p_{\mathrm{v}} .
$$

The compressional sound speed $c_{\mathrm{P}}$ and the density $\rho_{\mathrm{s}}$ refer to that of water-saturated sediment which surrounds the gas bubble and can be obtained from:

$$
c_{\mathrm{p}}=\sqrt{\frac{K_{\mathrm{s}}+4 G_{\mathrm{s}} / 3}{\rho_{\mathrm{s}}}},
$$

and

$$
\rho_{\mathrm{s}}=\xi \rho_{\mathrm{w}}+(1-\xi) \rho_{\mathrm{m}},
$$

respectively, where $K_{\mathrm{s}}$ is the bulk modulus of the water-saturated sediment, $\xi$ is the porosity, $\rho_{\mathrm{w}}$ is the water density and $\rho_{\mathrm{m}}$ the grain mineral density.

Berryman [64, 65] suggested a self-consistent method to calculate the effective bulk modulus of a composite medium, which states (as applied to the present problem)

$$
\begin{gathered}
\frac{1}{K_{\mathrm{s}}+4 G_{\mathrm{s}} / 3}=\frac{1-\xi}{K_{\mathrm{m}}+4 G_{\mathrm{s}} / 3}+\frac{\xi}{K_{\mathrm{w}}+4 G_{\mathrm{s}} / 3}, \\
\frac{1}{G_{\mathrm{s}}+F}=\frac{1-\xi}{G_{\mathrm{m}}+F}+\frac{\xi}{G_{\mathrm{w}}+F},
\end{gathered}
$$

where

$$
F=\left(\frac{G_{\mathrm{s}}}{6}\right) \frac{9 K_{\mathrm{s}}+8 G_{\mathrm{s}}}{K_{\mathrm{s}}+2 G_{\mathrm{s}}} .
$$

In (6) and (7), $K_{\mathrm{m}}$ refers to the bulk modulus of the solid matrix, $K_{\mathrm{w}}$ is the bulk modulus of pore water, $G_{\mathrm{m}}$ is the shear modulus of the solid matrix and $G_{\mathrm{w}}$ denotes the shear modulus of pore water which is related to the shear viscosity of water with $G_{\mathrm{w}}=i \omega \eta_{\mathrm{w}}$. 


\section{A. Thermal losses}

The Yang and Church model formulated the thermal processes within the bubble assuming an ideal gas with polytropic index $\kappa$ :

$$
p_{\mathrm{g}}=p_{\mathrm{g}_{0}}\left(\frac{R_{0}}{R}\right)^{3 \kappa} .
$$

If the value of $\kappa$ is constant throughout the bubble pulsation, the heat transfer across the bubble wall is reversible, and the above model does not predict any irreversible heat transfer (Leighton et al. [54]).

Prosperetti et al. [67] established a nonlinear energy conservation based formulation for the thermal losses during the expansion and compression of a spherical gas bubble. It was shown that, in the linear limit, these irreversible heat losses can be modelled with

$$
p_{\mathrm{g}}=p_{\mathrm{g}_{0}}\left(\frac{R_{0}}{R}\right)^{3 \kappa(\omega)}-4 \eta_{\mathrm{th}} \frac{\dot{R}}{R} \text {, }
$$

where $\eta_{\mathrm{th}}=\left(3 p_{\mathrm{g}_{0}} / 4 \omega\right) \operatorname{Im}(\Phi)$ is the "thermal viscosity" and $\omega$ is the angular frequency. According to Prosperetti et al. [67], the effective polytropic index $\kappa(\omega)$ is

$$
\kappa(\omega)=\frac{1}{3} \operatorname{Re}(\Phi),
$$

where the complex function

$$
\Phi=\frac{3 \gamma}{1-3(\gamma-1) i \chi\left[\left(\frac{i}{\chi}\right)^{\frac{1}{2}} \operatorname{coth}\left(\frac{i}{\chi}\right)^{\frac{1}{2}}-1\right]},
$$

which depends on the adiabatic polytropic exponent $\gamma$ and the thermal diffusivity of the gas $D_{\mathrm{g}}$ through the thermal diffusion length

$$
\chi=\frac{D_{\mathrm{g}}}{\omega R_{0}^{2}} .
$$


Although the use of a thermal viscosity to capture thermal losses remains approximate (there remains, for example, discussion as to the choice of frequency for use in the formulation when the bubble pulsation is not monochromatic), it gives a usefully simply analytical that avoids the complexities of calculating thermal losses from, say, maps of the time-dependent bubble volume response as a function of driving pressure [54].

\section{B. Viscoelastic constitutive relation}

The Voigt constitutive model used in the bubble dynamics model (2) can be expressed as:

$$
\tau=G_{\mathrm{s}} \varepsilon+\eta_{\mathrm{s}} \dot{\varepsilon},
$$

where $\varepsilon$ is the strain and $\dot{\varepsilon}$ is the strain rate. The Voigt model is appropriate for most marine sediments since the acoustic excitations induce small strains [20, 68, 69]. This model indicates that the total stress field in the medium will produce a superposition of both elastic and viscous responses (see schematic inset into Fig. 1). The common approach in terms of modelling the viscoelastic behaviour of marine sediments has been to treat the medium as a lossy elastic solid by using a complex shear modulus $[70,71]$ :

$$
G^{*}=G_{\mathrm{s}}+i G_{\mathrm{s}}^{\prime}
$$

The shear viscosity of the sediment is then deduced from the imaginary part of the shear modulus (equivalently from shear wave attenuation) [52, 69, 53, 72]:

$$
\eta_{\mathrm{s}}=\frac{G_{\mathrm{s}}^{\prime}}{2 \pi f}
$$

\section{LINEARIZATION}

To address the linear bubble dynamics problem, the resonance frequency and the linear damping terms will be derived from equation (2). Solutions to the linear problem can be found by assuming small amplitude pulsations $x \ll 1$ about the bubble equilibrium radius $R_{0}$, i.e. $R=R_{0}(1+x)$. Grouping the terms $x, \dot{x}$, and $\ddot{x}$ generates the following linearized formulation: 


$$
\ddot{x}+2 \beta_{\mathrm{tot}} \dot{x}+\omega_{0}^{2} x=-\frac{P_{A} e^{i \omega t}}{m_{\mathrm{eff}}},
$$

where $m_{\mathrm{eff}}$ is the effective mass

$$
m_{\text {eff }}=\rho_{\mathrm{s}} R_{0}^{2}+\frac{4 \eta_{\mathrm{s}} R_{0}}{c_{\mathrm{p}}} .
$$

The terms in equation (17) include the resonance frequency for the bubble wall displacement

$$
\omega_{0}^{2}=\left(3 p_{\mathrm{g}_{0}} \operatorname{Re}(\phi)-\frac{2 \sigma}{R_{0}}+4 G_{\mathrm{s}}\right) /\left(\rho_{\mathrm{s}} R_{0}^{2}+\frac{4 \eta_{\mathrm{s}} R_{0}}{c_{\mathrm{p}}}\right)
$$

and the term $\beta_{\text {tot }}$, which has units of (1/s) and is sum of the following five damping terms, namely the radiation, the viscous, the thermal, the interfacial and the elastic:

$$
\begin{gathered}
\beta_{\mathrm{vis}}=2 \eta_{\mathrm{s}} /\left(\rho_{\mathrm{s}} R_{0}^{2}+\frac{4 \eta_{\mathrm{s}} R_{0}}{c_{\mathrm{p}}}\right), \\
\beta_{\mathrm{P}-\mathrm{th}}=\left(\frac{3 p_{\mathrm{g}_{0}} \operatorname{Im}(\phi)}{2 \omega}\right) /\left(\rho_{\mathrm{s}} R_{0}^{2}+\frac{4 \eta_{\mathrm{s}} R_{0}}{c_{\mathrm{p}}}\right), \\
\beta_{\mathrm{rad}}=\frac{\rho_{\mathrm{s}} \omega^{2} R_{0}^{3}}{2 c_{\mathrm{p}}} /\left(\rho_{\mathrm{s}} R_{0}^{2}+\frac{4 \eta_{\mathrm{s}} R_{0}}{c_{\mathrm{p}}}\right), \\
\beta_{\text {int }}=-\sigma /\left(\rho_{\mathrm{s}} c_{\mathrm{p}} R_{0}^{2}+4 \eta_{\mathrm{s}} R_{0}\right), \\
\left.\beta_{\mathrm{el}}=2 G_{\mathrm{s}} /\left(\rho_{\mathrm{s}} c_{\mathrm{p}} R_{0}+4 \eta_{\mathrm{s}}\right)\right),
\end{gathered}
$$

where

$$
\beta_{\mathrm{tot}}=\beta_{\mathrm{vis}}+\beta_{\mathrm{P}-\mathrm{th}}+\beta_{\mathrm{ac}}+\beta_{\mathrm{int}}+\beta_{\mathrm{el}} .
$$

Note the features that differentiate the model established here from the one as applied to soft tissue by Yang and Church [66] are: (a) the interpretation of the viscoelastic model representing the bubble surrounding medium (i.e. the model parameters $G_{\mathrm{s}}$ and $\eta_{\mathrm{s}}$ ), (b) the interpretation of 
the parameters that are related to the host medium such as density and sound speed, and (c) the more comprehensive thermal damping which accounts for net heat losses.

\section{A. Sound speed and attenuation}

The complex sound speed in sediment containing gas bubbles can be derived from the linearized equation of bubble pulsations following the method outlined by Commander and Prosperetti [73]. Applicable to the problem considered here, the complex speed of sound in a gassy sediment, $c_{\mathrm{Pb}}$, is expressed as

$$
\frac{c_{\mathrm{p}}^{2}}{c_{\mathrm{Pb}}{ }^{2}}=1+4 \pi c_{\mathrm{p}}{ }^{2} \int_{0}^{\infty} \frac{R_{0} n\left(R_{0}\right)}{\omega_{0}^{2}-\omega^{2}+2 i \beta_{\mathrm{tot}} \omega} \mathrm{d} R_{0},
$$

where $n\left(R_{0}\right) \mathrm{d} R_{0}$ is the number of bubbles per unit volume with radii between $R_{0}$ and $R_{0}+$ $\mathrm{d} R_{0}$. Note that $\beta_{\text {tot }}$ includes the elastic damping and the interfacial damping in addition to the other damping mechanisms for bubbles in water. Setting $c_{\mathrm{p}} / c_{\mathrm{Pb}}=u-i v$ yields the expressions for phase velocity $V$ [73]

$$
V=c_{\mathrm{p}} / u
$$

and attenuation $A$ in $\mathrm{dB} / \mathrm{m}$

$$
A=8.6859\left(\omega v / c_{\mathrm{p}}\right)
$$

\section{APPLICATION OF THE MODEL TO GASSY SEDIMENTS}

In this section, the model established in Section I and II will be applied to gassy sediments by defining appropriate model input parameters. In shallow marine sediments, the gases that are most likely to be encountered are: oxygen $\left(\mathrm{O}_{2}\right)$, carbon dioxide $\left(\mathrm{CO}_{2}\right)$, nitrogen $\left(\mathrm{N}_{2}\right)$, ammonia $\left(\mathrm{NH}_{3}\right)$, hydrogen sulphide $\left(\mathrm{H}_{2} \mathrm{~S}\right)$ and methane $\left(\mathrm{CH}_{4}\right)$. Their production is mainly controlled by biogeochemical processes associated with the bacterial remineralisation of labile organic matter [74]. Although in the top sediment layer more than one gas may exist, the dominant ones are oxygen and aerobic respiration products in the aerobic zone, sulphate (and sulphur compounds) 
in the sulphate-reducing zone and methane in the carbonate reducing zone. Nitrogen is not a major gas in these zones; hence, $\mathrm{O}_{2}$ is assumed to be the predominant gas.

The density of pore water of seafloor sediments is nearly constant in marine environments and takes values between 1000 and $1030 \mathrm{~kg} \mathrm{~m}^{-3}$ [40]. The mineral density depends on the sediment type. Sands typically comprise quartz minerals with density equal to $2650 \mathrm{~kg} \mathrm{~m}^{-3}$, whereas muds are comprised of various minerals (kaolinite, illite etc.) having densities from $2600 \mathrm{~kg} \mathrm{~m}^{-}$ ${ }^{3}$ to $2800 \mathrm{~kg} \mathrm{~m}^{-3}$ (Mavko et al. [75]). In marine sediments, the porosity varies between 0.35 and 0.90 and is generally inversely proportional to the mean grain size [27].

The model values in line with these considerations are given in Table I. The value of the surface tension is difficult to determine and not available in literature; it is assumed to take the value for a bubble in salty water (no allowance being made for the level of dirtiness in seawater).

The bubble linear frequency is dominated by the shear modulus. In Fig. 1, the bubble resonance frequency is plotted against the bubble size using equation (16) (solid line), where it is shown that it varies with the square root of the shear modulus. On the same figure, the resonance frequency for the same bubble sizes in water (dotted line) is plotted for comparison.

Table I: Model input parameters:

\begin{tabular}{|c|l|l|c|l|}
\hline Property & Value & & Property & Value \\
\hline water depth & $1 \mathrm{~m}$ & & $K_{\mathrm{m}}$ & $5 \times 10^{10} \mathrm{~Pa}$ \\
\hline sediment depth & $1 \mathrm{~m}$ & & $G_{\mathrm{m}}$ & $1.2 \times 10^{7} \mathrm{~Pa}$ \\
\hline$p_{0}$ & $101325 \mathrm{~Pa}$ & & $G_{\mathrm{s}}^{\prime}$ & $0.16 \mathrm{MPa}$ \\
\hline$\rho_{\mathrm{w}}$ & $998 \mathrm{~kg} / \mathrm{m}^{3}$ & & $\kappa$ & 1.4 \\
\hline$\rho_{\mathrm{s}}$ & $1640 \mathrm{~kg} / \mathrm{m}^{3}$ & & $\sigma$ & $0.036 \mathrm{~N} / \mathrm{m}$ \\
\hline$c_{\mathrm{p}}$ & $1465 \mathrm{~m} / \mathrm{s}$ & & $\rho_{\mathrm{g}}$ & $1.202 \mathrm{~kg} / \mathrm{m}^{3}$ \\
\hline$K_{\mathrm{w}}$ & $2.3 \mathrm{GPa}$ & & $K_{\mathrm{g}}$ & $0.0241 \mathrm{~W} / \mathrm{mK}$ \\
\hline$\eta_{\mathrm{w}}$ & $0.0018 \mathrm{~Pa} \mathrm{~s}$ & & $C_{\mathrm{pg}}$ & $1001.73 \mathrm{~J} / \mathrm{kgK}$ \\
\hline$\xi$ & 0.68 & & $D_{\mathrm{g}}$ & $1.9 \times 10^{-5} \mathrm{~m}^{2} / \mathrm{s}$ \\
\hline
\end{tabular}




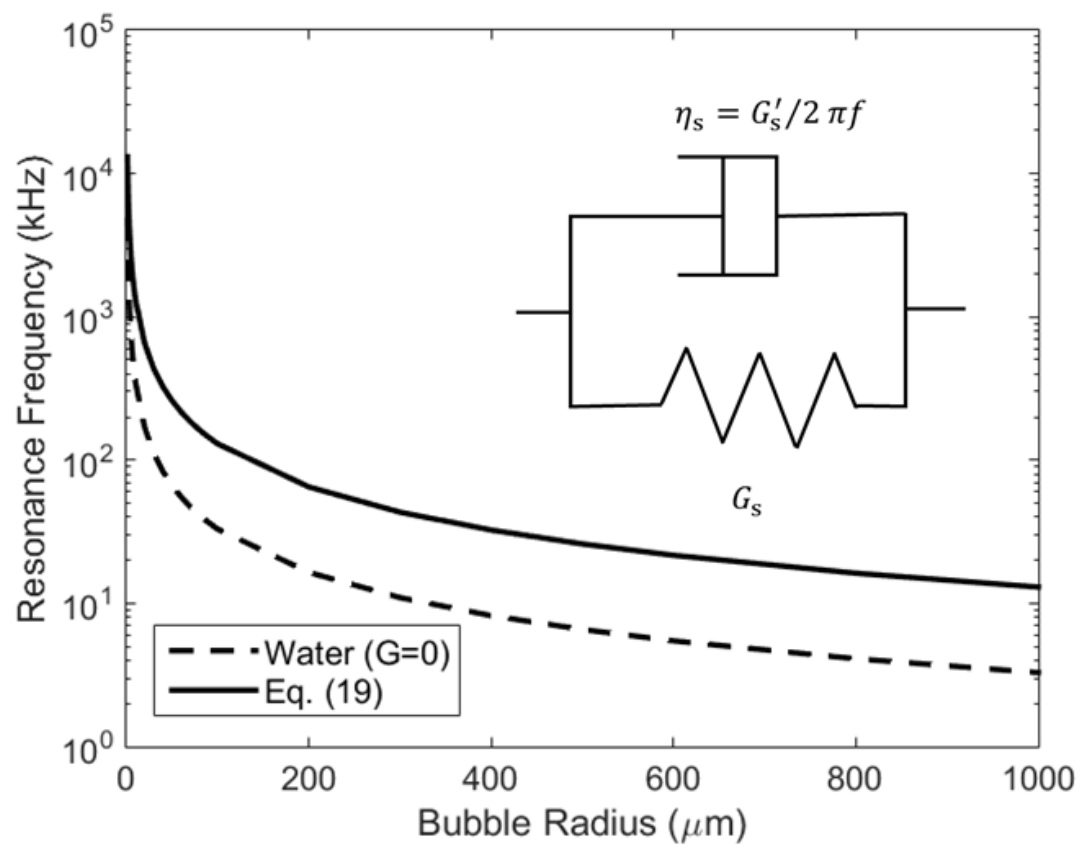

Figure 1: Bubble resonance frequency vs. bubble size $\left(R_{0}\right)$. The solid line is calculated by equation (19) and the dashed line is calculated from the same equation by setting zero shear modulus. The input parameters for both cases are shown in Table I. The inset shows a schematic of a mechanical spring-dashpot analogue representing the responses of rheological elementary model: the Voigt model with constant parameters. The shear viscosity for sediments is deduced from the imaginary part of the shear modulus, i.e. $\eta_{\mathrm{s}}=G_{\mathrm{s}}^{\prime} / 2 \pi f$.

Next, the results for the damping terms are presented in Fig. 2. The results indicate that the acoustic radiation damping is the dominant loss mechanism at high frequencies, justifying the extension suggested in [20] to include these. An equally important loss mechanism appears to be the viscous damping as formulated by equation (20a). The elastic damping, which was not identified in the Anderson and Hampton theory but is formulated here owing to the complete form of the Voigt constitutive model used, is also a significant damping mechanism as can be seen. The effects of the thermal damping are relatively less pronounced and the interfacial damping can be regarded as negligible. However, we should remark that the thermal damping may be a dominant loss mechanism at medium frequencies (according to the range in the figure) depending on the sediment type investigated and the gas content of the medium (e.g. in muddy sediments where rigidity values are much lower and the sediment resembles more fluidlike behaviour). Note that the thermal damping based on the formulation of Prosperetti et al. 
[67] (labelled as P-thermal) shows qualitatively different behaviour as the frequency changes. The thermal damping according to ideal gas law (9) does not show frequency dependent behaviour and does not predict any net thermal losses over a cycle if the polytropic exponent is taken as constant [54]. Overall, the damping characteristics stemming from the elastic properties of the host medium are very significant, indicating that models which have treated the host sediment as water will underestimate the damping.
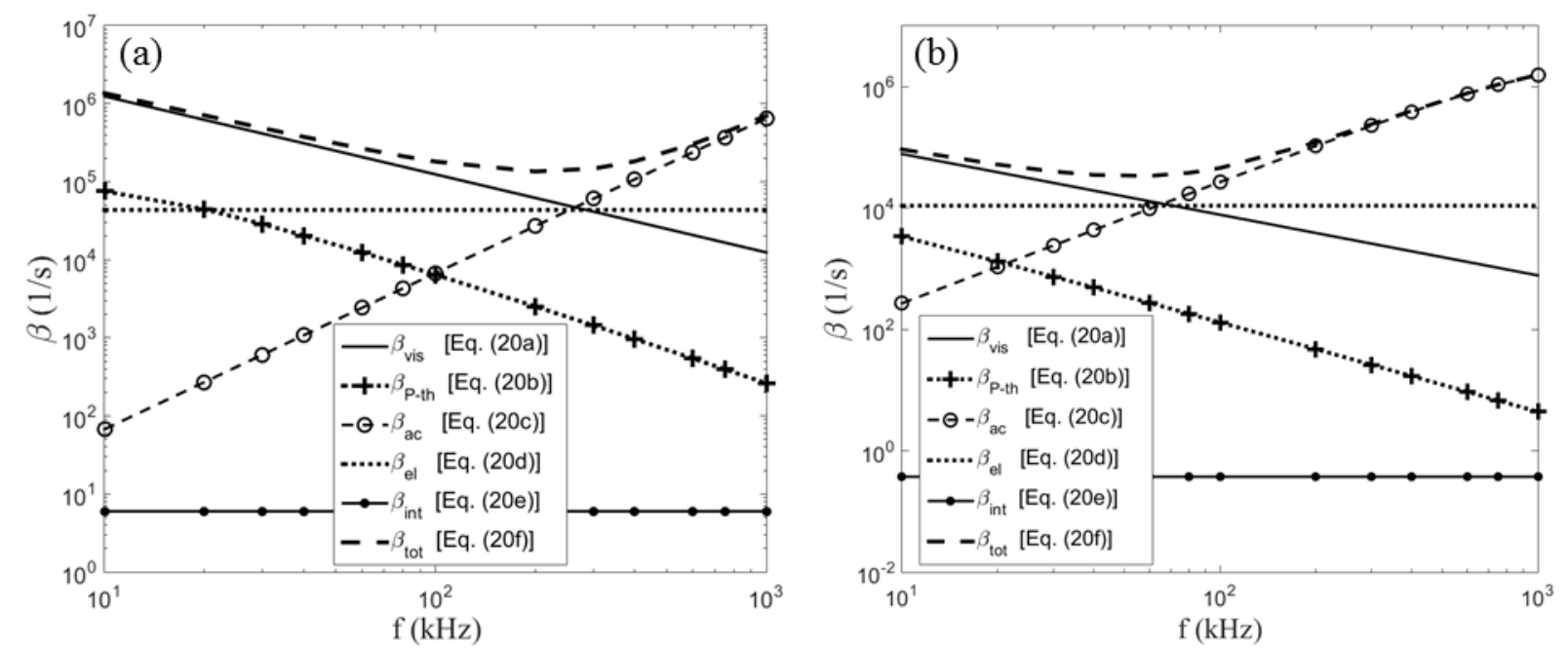

Figure 2: Dimensional damping constants vs. frequency for a bubble with equilibrium radius (a) $50 \mu \mathrm{m}$ and (b) $200 \mu \mathrm{m}$ for the parameters listed in Table I.

Using equations (22)-(23), the sound speed and attenuation of an acoustic wave has been computed for monodiosperse bubble populations of radii 80, 120, 160 and $200 \mu \mathrm{m}$, assuming the input parameters of Table I. The results are shown in Fig. 3 (solid line). On the same figure the sound speed and attenuation are plotted (dotted line) using equations 49 and 53 of the Anderson and Hampton model [52]. The difference in the predictions of these two models is attributed to the differences in the damping terms and the linearization of the fundamental equation used (2). The results point out similarities at low frequencies and at high frequencies: in the former case, the propagation velocities in the medium is largely determined by the gas void fraction and the bulk modulus of the gas, and in the latter case the sound speed converges to that of water saturated medium. The near resonance regime behaviour of the models, on the other hand, is significantly different. Given that the present results involve single bubble size, it is anticipated the predictions differ even more in case a bubble size distribution is attempted to be modelled/inverted. As already discussed, the main advantage of equation (22) is that it does 
not contain sign ambiguity and it can be used in an inversion model which requires simultaneous information on both sound speed and attenuation data, as in Duraiswami et al. [76]. In line with the near resonance sound speed results, the attenuation data (fig. 3b) shows that the present model predicts higher attenuation for single bubble sizes considered.
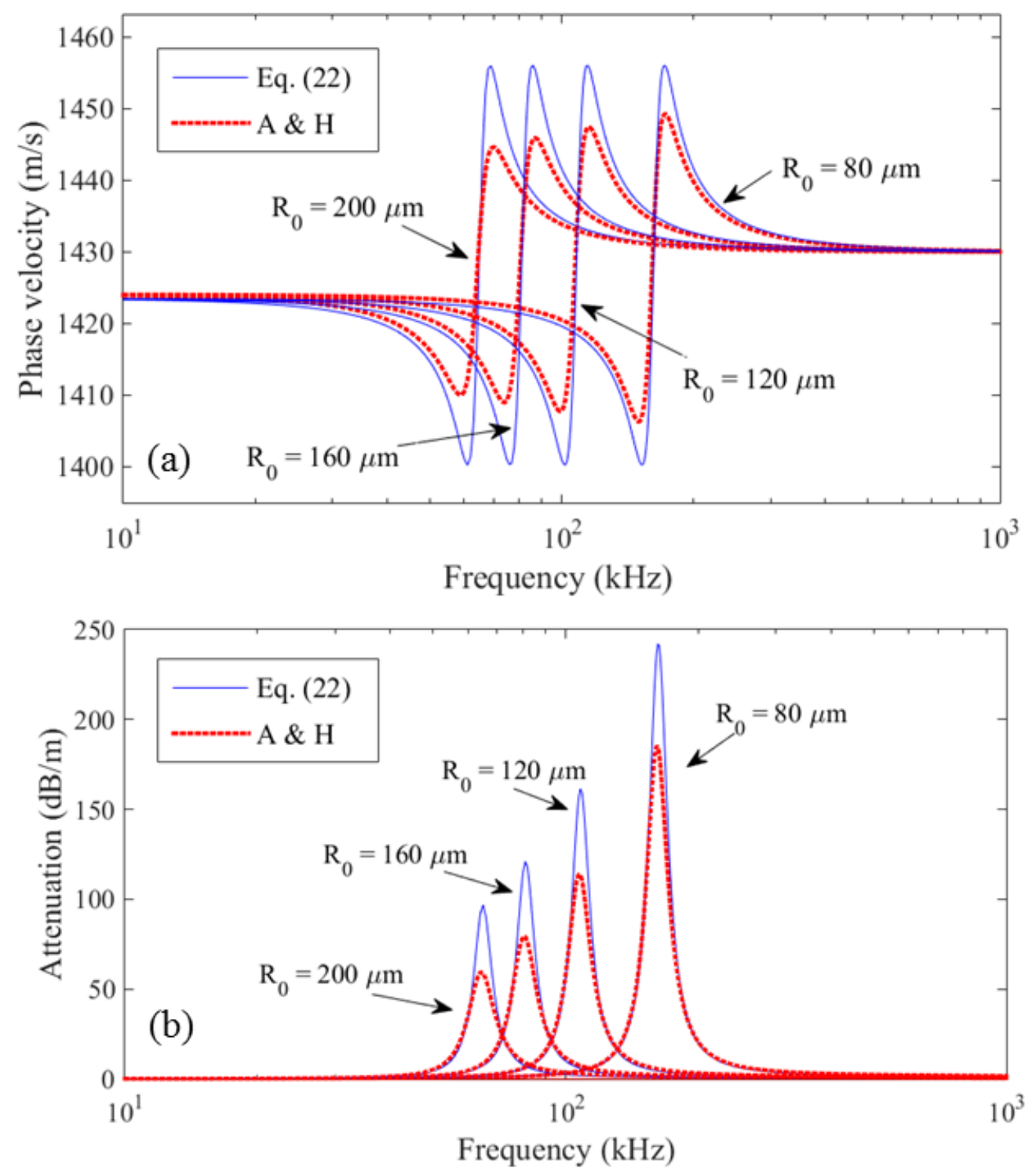

Figure 3: (a) Sound speed and (b) Attenuation as function of frequency for a series of monodisperse bubble populations, all having a void fraction of $10^{-5}$ (the bubble radius for each being labelled on the figure). The dashed line shows the predictions of the Anderson and Hampton model [52] (A\&H), and the solid line shows the results of the current formulation. 


\section{NONLINEAR SCATTERING}

The scattering cross section may be defined as the ratio of the total acoustic power scattered by an object at a given frequency to the incoming acoustic intensity $I_{0}$ :

$$
\sigma_{\mathrm{s}}=\frac{4 \pi r^{2} I_{\mathrm{s}}}{I_{0}}
$$

where $I_{0}=\left|P_{\mathrm{A}}\right|^{2} / 2 \rho_{\mathrm{S}} c_{\mathrm{S}}$ and $I_{\mathrm{S}}=\left|P_{\mathrm{b}}\right|^{2} / 2 \rho_{\mathrm{S}} c_{\mathrm{S}}$. To calculate the nonlinear scattering, the pressure $P_{\mathrm{b}}$ radiated from a single bubble is obtained from [58]:

$$
P_{b}(r, t)=\frac{\rho_{\mathrm{s}}}{r}\left(2 R \dot{R}^{2}+R^{2} \ddot{R}\right)
$$

If linear pulsations of bubbles are considered, the above equations reduce to:

$$
\sigma_{\mathrm{s}}=\frac{4 \pi R_{0}^{2}}{\left(\omega^{2} / \omega_{0}^{2}-1\right)^{2}+4 \beta_{\mathrm{tot}}^{2} / \omega^{2}}
$$

Furthermore, applicable to both linear and nonlinear scattering, when a bubble distribution is present, the scattering cross section per unit volume is:

$$
\sigma_{\mathrm{v}}=\frac{1}{4 \pi} \int_{0}^{\infty} n_{b}\left(R_{0}\right) \sigma_{\mathrm{s}} d R_{0},
$$

where $n_{\mathrm{b}}\left(R_{0}\right)$ is the bubble number density (as defined in Section II.A), which is typically expressed in terms of discrete radius bins, e.g. with $1 \mu \mathrm{m}$ width. In practice, the limits of the integration are determined by the minimum and maximum detectable bubble size, which are deduced from the excitation frequency range through the use of equation (19). The volume backscattering strength $\left(S_{\mathrm{v}}\right)$ of a bubble population is defined with the reference volume cross section $\sigma_{\mathrm{v} \text { ref }}=1 \mathrm{~m}^{2} / \mathrm{m}^{3}$ : 
$S_{\mathrm{v}}=10 \log _{10}\left(\sigma_{\mathrm{v}} / \sigma_{\mathrm{v} \text { ref }}\right)$.

The difference between the present model and Anderson and Hampton's theory is demonstrated by means of applying equations (24) and (25), and equation (26), respectively. The results are shown in Fig. 4, the input bubble size distribution for the calculation being shown in the inset. The present model predicts lower values of the backscattering strength owing to the fundamental differences of the formulations. Although the steady-state responses are shown in Fig. 4, the current model is capable of modelling transient behaviour such as ring-up and ringdown periods during the insonification.

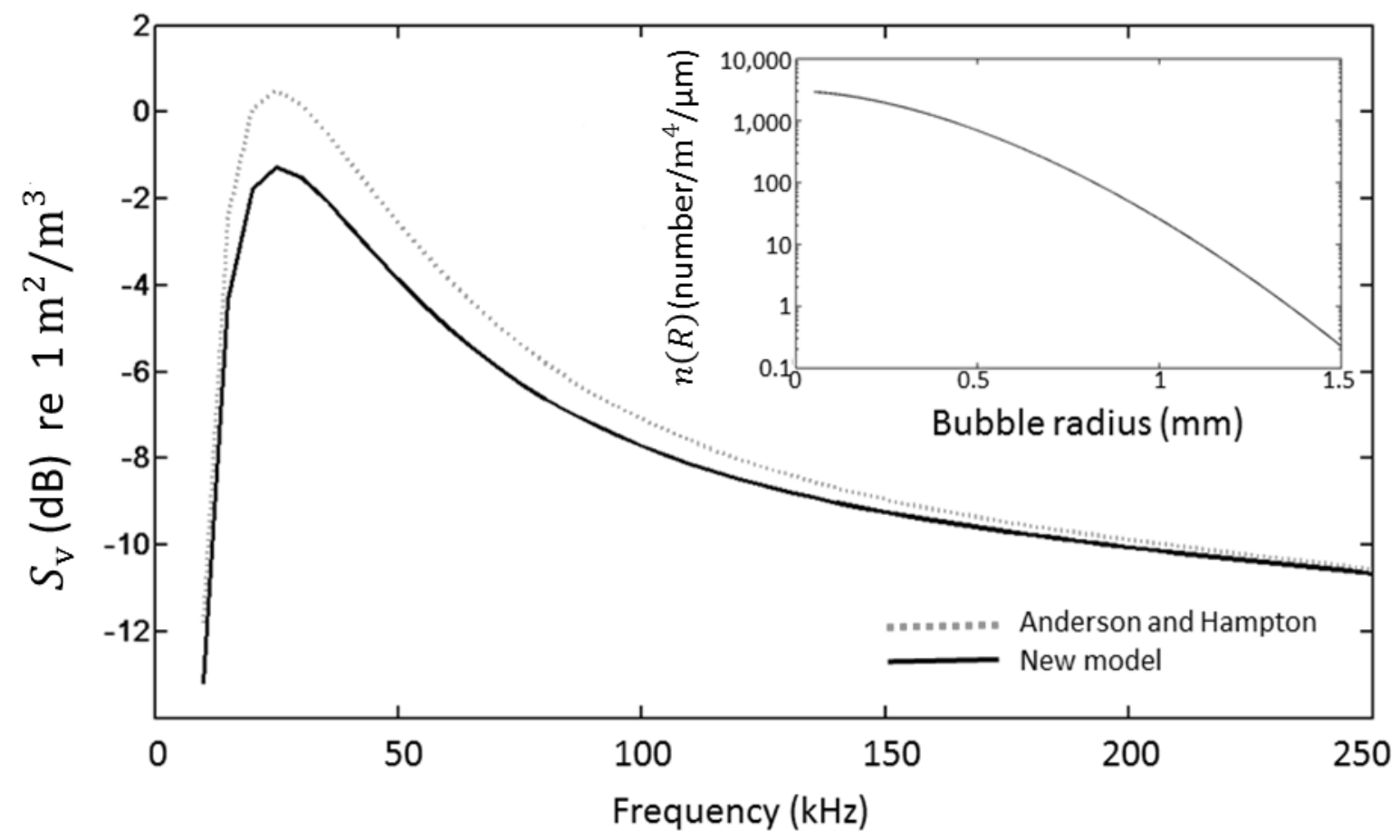

Figure 4: Volume backscattering strength (defined with the reference volume cross section $\sigma_{\mathrm{v} \text { ref }}=1 \mathrm{~m}^{2} / \mathrm{m}^{3}$ ) vs. the frequency, corresponding to the bubble size distribution (BSD) shown in the inset. The scattering cross-section expressions are used according to the Anderson and Hampton formulation (dotted line) and the present model (solid line). The inset BSD has a void fraction of $2.26 \times 10^{-4}$.

Note that the nonlinearity referred to in this section manifests itself in two distinct fashions. The first one is the nonlinear pulsations of gas bubbles as demonstrated above, and the second one is the nonlinear mixing of frequency components when dual frequency excitation 
techniques are employed. The majority of the studies which used dual frequency techniques derived analytical expressions for the scattered pressure spectral component at the differencefrequency, e.g. see Refs. [17, 63, 62, 16]. An alternative approach [77] suggests that the cumbersome scattering cross-section expressions encountered in dual frequency excitation can be avoided by numerically calculating the time history of the bubble radius (2), inserting these values into (25) to obtain the scattered pressure field, and applying a Fourier transform to determine the spectral components of the scattered pressure. The pressure spectral amplitudes are then compared to the recorded experimental data at each frequency, which yields the determination of the number of bubbles per unit volume. This numerical approach has been applied for bubble sizing in water tank experiments in Leighton et al. [77] and in sediments by Mantouka [78].

An example numerical computation of the spectral amplitude is shown in Fig. 5. The nonlinear volume backscattering strength at twice the insonification frequency is plotted versus the insonification frequency for the bubble distribution shown in the inset of Fig. 4. First, $R, \dot{R}$ and $\ddot{R}$ were computed from equation (2) assuming a monochromatic incident wave $P_{A} e^{i \omega t}$, and substituted into equation (25) to calculate the scattered pressure $P_{b}(t)$. The power spectra of the time series of $P_{b}$ were calculated using a Fourier transform, and the spectral component nearest twice the insonification frequency is then used in Eq. (28) (using normalized values). Only bubbles resonant at the insonification frequency give strong nonlinear scattering at twice the frequency. Hence the beginning of the curve corresponds to the first frequency where the bubbles of 50 microns are resonant (which is the assumed small-bubble limit of the bubble distribution in the simulation). 


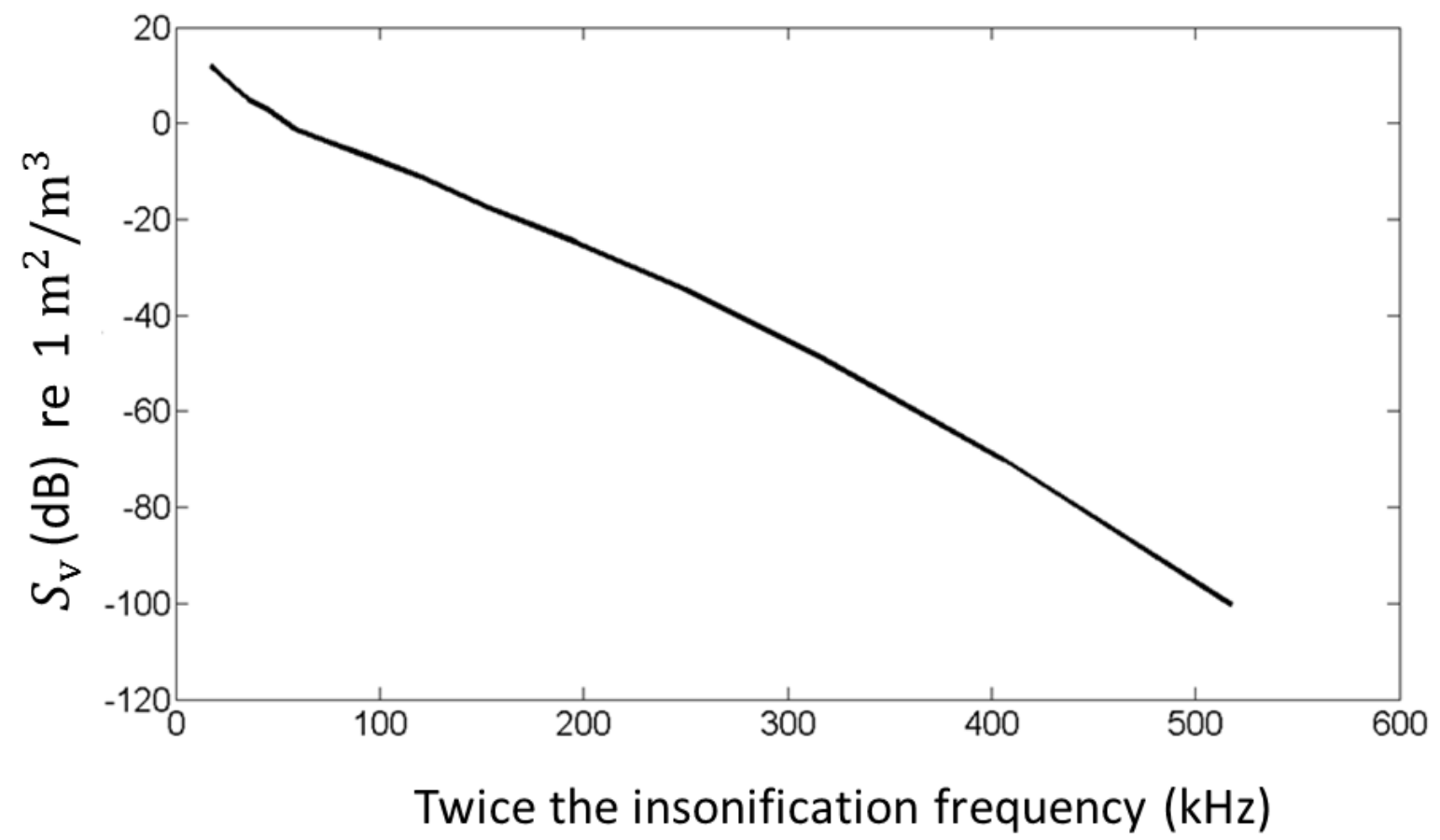

Figure 5: The nonlinear volume backscattering strength at twice the insonification frequency (defined with the reference volume cross section $\sigma_{\mathrm{v} \text { ref }}=1 \mathrm{~m}^{2} / \mathrm{m}^{3}$ ).

\section{DISCUSSION AND CONCLUSIONS}

This paper has presented a nonlinear model for gas bubble pulsations in marine sediments. The expressions are linearized to determine the resonance frequency and the damping terms for linear bubble pulsations, and then used to predict the effects that such bubble pulsations will have on the sound speed and attenuation of acoustic waves propagating in gassy marine sediment. The results between the current model and an existing theory (Anderson and Hampton) show significant differences at near resonance regimes. One major advantage of the present model is that, without user intervention to overcome the sign ambiguity, it can automatically produce objective prediction of sound speed and attenuation, and invert measurements of these parameters to infer the bubble population. Furthermore, the bubble pulsation model (2) can be used to predict the amplitudes of the scattered pressure field at the nonlinearly generated frequency components, i.e. the second harmonics, and combination and difference frequencies. Moreover, it can be included into a scheme to predict the effect on the sound speed and attenuation when the bubbles pulsate in a nonlinear manner (Leighton et al. 
[54]). In this work, the formulation is proposed as a forward model and an improved alternative to the existing theory for gassy soft marine sediments. The inversion of real acoustic experimental data and the obtained bubble population results will be subject of a follow-up article.

As a preliminary analysis, the present formulation assumed the propagation of one compressional wave in the host medium (the far field pressure in existing theories is modelled with a single velocity potential without losses). Note that the work of Mantouka [78] heuristically accounted for the attenuation of the compressional wave, by assuming the forward model as in the present article but adding the corrections from the internal dissipation at the inversion stage (for bubble populations). A more comprehensive approach is required to incorporate the complex characteristics of the acoustic propagation in porous medium, i.e. the propagation of the second mode (slow wave), and the attenuation of the longitudinal modes due to internal dissipation, squirt flow and shear drag [79]. The addition of gas bubbles into the problem thereafter can be done by either modifying the radial dynamics equation (2) or extending the formulation in Kargl et al. [48].

\section{ACKNOWLEDGEMENTS}

This work is funded in part by the Natural Environment Research Council (NERC, RP008255) and the Engineering and Physical Sciences Research Council (EP/D000580/1; Principal Investigator: T. G. Leighton) and STEMM-CCS (funded from the European Union's Horizon 2020 research and innovation programme under grant agreement No. 654462). Agni Mantouka's PhD studentship was supported by ISVR's Rayleigh Scholarship Scheme. The authors wish to thank Dr Gary Heald, Dr Angus Best, Dr Paul Fox and Dr Justin Dix for useful discussions. Hakan Dogan's contributions were supported by NERC (NE/J022403/1; Principal Investigator: T. G. Leighton). 


\section{FIGURE CAPTIONS}

Figure 1: Bubble resonance frequency vs. bubble size $\left(R_{0}\right)$. The solid line is calculated by equation (19) and the dashed line is calculated from the same equation by setting zero shear modulus. The input parameters for both cases are shown in Table I. The inset shows a schematic of a mechanical spring-dashpot analogue representing the responses of rheological elementary model: the Voigt model with constant parameters. The shear viscosity for sediments is deduced from the imaginary part of the shear modulus, i.e. $\eta_{\mathrm{s}}=G_{\mathrm{s}}^{\prime} / 2 \pi f$.

Figure 2: Dimensional damping constants vs. frequency for a bubble with equilibrium radius (a) $50 \mu \mathrm{m}$ and (b) $200 \mu \mathrm{m}$ for the parameters listed in Table I.

Figure 3: (a) Sound speed and (b) Attenuation as function of frequency for a series of monodisperse bubble populations, all having a void fraction of $10^{-5}$ (the bubble radius for each being labelled on the figure). The dashed line shows the predictions of the Anderson and Hampton model [52] (A\&H), and the solid line shows the results of the current formulation.

Figure 4: Volume backscattering strength (defined with the reference volume cross section $\sigma_{\mathrm{v} \text { ref }}=1 \mathrm{~m}^{2} / \mathrm{m}^{3}$ ) vs. the frequency, corresponding to the bubble size distribution (BSD) shown in the inset. The scattering cross-section expressions are used according to the Anderson and Hampton formulation (dotted line) and the present model (solid line). The inset BSD has a void fraction of $2.26 \times 10^{-4}$.

Figure 5: The nonlinear volume backscattering strength at twice the insonification frequency (defined with the reference volume cross section $\sigma_{\mathrm{v} \text { ref }}=1 \mathrm{~m}^{2} / \mathrm{m}^{3}$ ). 


\section{REFERENCES}

[1] G. C. Sills and S. J. Wheeler, "The significance of gas for offshore operations," Continental Shelf Research, vol. 12, no. 10, pp. 1239-1250, 1992.

[2] G. C. Sills, S. J. Wheeler, S. D. Thomas and T. N. Gardiner, "Behaviour of offshore soils containing gas bubbles," Geotechnique, vol. 41, pp. 227-241, 1991.

[3] K. B. Briggs and M. D. Richardson, "Variability in in situ shear strength of gassy muds," Geo-Mar. Lett. , vol. 16, pp. 189-195, 1996.

[4] T. G. Leighton and P. R. White, "Quantification of undersea gas leaks from carbon capture and storage facilities, from pipelines and from methane seeps, by their acoustic emissions," Proceedings of the Royal Society A - Mathematical, Physical and Engineering Sciences, vol. 468, pp. 485-510, 2012.

[5] E. J. Sauter, S. I. Muyakshin, J.-L. Charlou, M. Schlüter, A. Boetius, K. Jerosch, E. Damm, J.-P. Foucher and M. Klages, "Methane discharge from a deep-sea submarine mud volcano into the upper water column by gas hydrate-coated methane bubbles," Earth Planet Science Letters, vol. 243, pp. 354-365, 2006.

[6] I. Leifer, D. Roberts, J. Margolis and F. Kinnaman, "In situ sensing of methane emissions from natural marine hydrocarbon seeps: a potential remote sensing technology," Earth Planet. Sci. Lett., vol. 245, pp. 509-522, 2006.

[7] A. I. Best, M. D. Richardson, B. P. Boudreau, A. G. Judd, I. Leifer, A. P. Lyons, C. S. Martens, D. L. Orange and S. J. Wheeler, "Shallow bed methane gas could pose coastal hazard,” EOS Transactions, vol. 87, no. 22, pp. 213-217, 2006.

[8] J. Blackford, H. Stahl, J. Bull and et al, "Detection and impacts of leakage from subseafloor deep geological carbon dioxide storage," Nature Climate Change, vol. 4, pp. 1011-1016, 2014.

[9] J. Blackford, J. M. Bull, M. Cevatoglu, D. Connelly, C. Hauton, R. H. James, A. Lichtschlag, H. Stahl, S. Widdicombe and I. C. Wright, "Marine baseline and monitoring strategies for Carbon Dioxide Capture and Storage (CCS)," International Journal of Greenhouse Gas Control, vol. 38, pp. 221-229, 2015.

[10] B. J. P. Berges, T. G. Leighton and P. R. White, "Passive acoustic quantification of gas fluxes during controlled gas release experiments," International Journal of Greenhouse Gas Control, vol. 38, pp. 64-79, 2015.

[11] T. G. Leighton and G. Robb, "Preliminary mapping of void fractions and sound speeds in gassy marine sediments from subbottom profiles," Journal of the Acoustical Society of 
America, vol. 124, no. 5, pp. EL313-EL320, 2008.

[12] G. B. N. Robb, S. P. Robinson, P. D. Theobald, G. Hayman, V. F. Humphrey, T. G. Leighton, L. S. Wang, J. K. Dix and A. I. Best, "Absolute calibration of hydrophones immersed in sandy sediment,” J. Acoust. Soc. Am., vol. 125, no. 5, pp. 2918-2927 , 2009.

[13] T. G. Leighton, A. D. Phelps, D. G. Ramble and D. A. Sharpe, "Comparison of the abilities of eight acoustic techniques to detect and size a single bubble," Ultrasonics, vol. 34, pp. 661-667, 1996.

[14] E. A. Zabolotskaya and S. I. Soluyan, "Emission of harmonic and combination frequency waves by air bubbles,” Sov. Phys. Acoust., vol. 18, pp. 396-398, 1973.

[15] T. G. Leighton, D. G. Ramble and A. D. Phelps, "The detection of tethered and rising bubbles using multiple acoustic techniques,” J. Acoust. Soc. Am. , vol. 101, pp. 2626-2635, 1997.

[16] S. V. Karpov, Z. Klusek, A. L. Matveev, A. I. Potapov and A. M. Sutin, "Nonlinear interaction of acoustic wave in gas-saturated marine sediments," Acoustical Physics , vol. 42, pp. 527-533 , 1996.

[17] F. A. Boyle and N. P. Chotiros, "Nonlinear acoustic scattering from a gassy poroelastic seabed,” J. Acoust. Soc. Am., vol. 103, pp. 1328-1336, 1998.

[18] Z. Klusek, A. Sutin, A. Matveev and A. Potapov, "Observation of nonlinear scattering of acoustical waves at sea sediments,” Acoustical Letters , vol. 18, pp. 198-203, 1995.

[19] T. G. Leighton, “The Rayleigh-Plesset equation in terms of volume with explicit shear losses,” Ultrasonics, vol. 48, pp. 85-90, 2008.

[20] T. G. Leighton, “Theory for acoustic propagation in marine sediment containing gas bubbles which may pulsate in a non-stationary nonlinear model,” Geophysical Research Letters , vol. 34, p. L17607, 2007.

[21] E. L. Hamilton, “Compressional wave attenuation in marine sediments,” Geophysics, vol. 4, pp. 620-646, 1972.

[22] S. S. Fu, R. H. Wilkens and L. N. Frazer, "In situ velosity profiles in gassy sediments: Kiel Bay,” Geo-Marine Letters, vol. 16, pp. 249-253, 1996.

[23] M. D. Richardson and K. B. Briggs, "In situ and laboratory geoacoustic measurements in soft mud and hard-packed sand sediments: Implications for high-frequency acoustic propagation and scattering," Geo-Marine Letters, vol. 16, pp. 196-203, 1996. 
[24] G. B. N. Robb, A. I. Best, J. K. Dix, J. M. Bull, T. G. Leighton and P. R. White, "The frequency dependence of compressional wave velocity and attenuation coefficient of intertidal marine sediments,” J. Acoust. Soc. Am. , vol. 120, pp. 2526-2537, 2006.

[25] E. L. Hamilton, G. Shumway, H. W. Menard and C. J. Shipek, "Acoustics and other physical properties of shallow-water sediments off San Diego," J. Acoust. Soc. Am., vol. 28, pp. 1-15, 1956.

[26] E. L. Hamilton, "Attenuation of shear waves in marine sediments," J. Acoust. Soc. Am., vol. 60, pp. 334-338, 1976.

[27] E. L. Hamilton and R. T. Bachman, "Sound velocity and related properties of marine sediments,” J. Acoust. Soc. Am., vol. 72, pp. 1891-1904, 1982.

[28] G. Shumway, "Sound speed and absorption studies of marine sediment by a resonance method," Geophysics, vol. 25, pp. 451-467, 1960.

[29] G. Shumway, "Sound speed and absorption studies of marine sediment by a resonance method-Part II,” Geophysics , vol. 25, pp. 659-681, 1960.

[30] A. I. Best, Q. J. Huggett and A. J. K. Harris, "Comparison of in situ and laboratory acoustic measurementson Lough Hyne marine sediments," J. Acoust. Soc. Am., vol. 110, pp. 695-709, 2001.

[31] J. Yang, D. Tang and K. L. Williams, "Direct measurement of sediment sound speed in ShallowWater ’06,” J. Acoust. Soc. Am. , vol. EL 124, pp. 116-121, 2008.

[32] A. Turgut and T. Yamamoto, "Measurements of acoustic wave velocities and attenuations in marine sediment,” J. Acoust. Soc. Am., vol. 87, pp. 2376-2383, 1990.

[33] G. Robb, A. Best, J. Dix, P. White, T. Leighton, J. Bull and A. Harris, "The measurement of the in situ compressional wave properties of marine sediments," IEEE Journal of Oceanic Engineering, vol. 32, pp. 484-496, 2007.

[34] A. B. Wood and D. E. Weston, “The propagation of sound in mud,” Acustica, vol. 14, pp. 156-162, 1964.

[35] F. A. Bowles, "Observations on attenuation and shear-wave velocity in fine-grained marine sediments,” J. Acoust. Soc. Am., vol. 101, pp. 3385-3397, 1997.

[36] R. D. Stoll, "Experimental studies of attenuation in sediments,” J. Acoust. Soc. Am., vol. 66, no. 4, pp. 1152-1160, 1979. 
[37] B. A. Brunson and R. K. Johnson, "Laboratory measurements of shear wave attenuation in saturated sand,” J. Acoust. Soc. Am. , vol. 68, pp. 1371-1375, 1980.

[38] M. A. Biot, "Theory of propagation of elastic waves in fluid-saturated porous solids: I. Low-frequency range,” J. Acoust. Soc. Am., vol. 28, no. 2, pp. 168-178, 1956.

[39] M. A. Biot, "Theory of propagation of elastic waves in fluid-saturated porous solids: II. Higher frequency range,” J. Acoust. Soc. Am. , vol. 28, no. 2, pp. 179-191, 1956.

[40] R. D. Stoll, "Marine sediment acoustics,” J. Acoust. Soc. Am., vol. 77, no. 5, pp. 17891799, 1985.

[41] J. G. Berryman, “Confirmation of Biot's theory,” Appl. Phys. Lett., vol. 37, no. 4, pp. 382384, 1980.

[42] K. L. Williams, D. R. Jackson, E. I. Thorsos and D. Tang, "Comparison of sound speed and attenuation measured in a sandy sediment to predictions based on the Biot theory of porous media,” IEEE J. Ocean. Eng., vol. 27, pp. 413-428, 2002.

[43] D. R. Jackson and M. D. Richardson, High-frequency Seafloor Acoustics, New York: Springer, 2007, pp. 1-616.

[44] J. A. Hawkins and A. Bedford, "Variational theory of bubbly media with a distribution of bubble sizes. 2: Porous solids,” Int. J. Eng. Sci., vol. 30, no. 9, pp. 1177-1186, 1992.

[45] J. L. Buchanan, “A comparison of broadband models for sand sediments," J. Acoust. Soc. Am., vol. 120, no. 6, pp. 3584-3598, 2006.

[46] D. M. J. Smeulders and M. E. H. Van Dongen, "Wave propagation in porous media containing a dilute gas-liquid mixture,” J. Fluid Mech. , vol. 343, pp. 351-373, 1997.

[47] A. L. Anderson, F. Abegg, J. A. Hawkins, M. E. Duncan and A. P. Lyons, "Bubble populations and acoustic interaction with the gassy floor of Eckernforde Bay," Continental Shelf Research, vol. 18, pp. 1807-1838, 1998.

[48] S. G. Kargl, K. L. Williams and R. Lim, "Double monopole resonance of a gas-filled, spherical cavity in a sediment,” J. Acoust. Soc. Am., vol. 103, no. 1, pp. 265-274, 1998.

[49] F. A. Boyle and N. P. Chotiros, "A model for acoustic backscatter from muddy sediments,” J. Acoust. Soc. Am., vol. 98, no. 1, pp. 525-530, 1995.

[50] F. A. Boyle and N. P. Chotiros, “A model for high-frequency acoustic backscatter from 
gas bubbles in sandy sediments at shallow grazing angles,” J. Acoust. Soc. Am., vol. 98, no. 1, pp. 531-541, 1995.

[51] A. L. Anderson and L. D. Hampton, “Acoustics of gas-bearing sediments I. Background,” J. Acoust. Soc. Am., vol. 67, no. 6, pp. 1865-1889, 1980.

[52] A. L. Anderson and L. D. Hampton, "Acoustics of gas-bearing sediment II. Measurements and models,” J. Acoust. Soc. Am., vol. 67, no. 6, pp. 1890-1903, 1980.

[53] A. I. Best, M. D. J. Tuffin,J. K. Dix and J. M. Bull, "Tidal height and frequency dependence of acoustic velocity and attenuation in shallow gassy marine sediments," Journal of Geophysical Research, vol. 109, no. B8, p. B08101, 2004.

[54] T. G. Leighton, S. D. Meers and P. R. White, "Propagation through nonlinear timedependent bubble clouds and the estimation of bubble populations from measured acoustic characteristics,” Proc. R. Soc. Lond. A, vol. 460, no. 2049, pp. 2521-2550, 2004.

[55] A. P. Lyons, M. E. Duncan and A. L. Anderson, "Predictions of the acoustic scattering response of free-methane bubbles in muddy sediments,” J. Acoust. Soc. Am., vol. 99, no. 1, pp. 163-172, 1996.

[56] M. A. Ainslie and T. G. Leighton, "Near resonant bubble acoustic cross-section corrections, including examples from oceanography, volcanology, and biomedical ultrasound,” J. Acoust. Soc. Am., vol. 126, no. 5, pp. 2163-2175, 2009.

[57] M. A. Ainslie and T. G. Leighton, "Review of scattering and extinction cross-sections, damping factors, and resonance frequencies of a spherical gas bubble,” J. Acoust. Soc. Am., vol. 130, no. 5, pp. 3184-3208, 2011.

[58] J. W. L. Clarke and T. G. Leighton, “A method for estimating time-dependent acoustic cross-sections of bubbles and bubble clouds prior to the steady state," Journal of the Acoustical Society of America, vol. 107, no. 4, pp. 1922-1929, 2000.

[59] A. D. Phelps, D. G. Ramble and T. G. Leighton, "The use of a combination frequency technique to measure the surf zone bubble population," Journal of the Acoustical Society of America, vol. 101, no. 4, pp. 1981-1989, 1997.

[60] A. D. Phelps and T. G. Leighton, "Oceanic bubble population measurements using a buoydeployed combination frequency technique,” IEEE J. Oceanic Eng., vol. 23, pp. 400-410, 1998.

[61] T. G. Leighton, R. J. Lingard, A. J. Walton and J. E. Field, “Acoustic bubble sizing by the combination of subharmonic emissions with an imaging frequency,” Ultrasonics, vol. 29, 
pp. 319-323, 1991.

[62] L. A. Ostrovsky, A. M. Sutin, I. A. Soustova, A. L. Matveyev, A. I. Potapov and Z. Kluzek, "Nonlinear scattering of acoustic waves by natural and artificially generated subsurface bubble layers in sea,” J. Acoust. Soc. Am., vol. 113, no. 2, pp. 741-749, 2003.

[63] A. M. Sutin, S. W. Yoon, E. J. Kim and I. N. Didenkulov, "Nonlinear acoustic method for bubble density measurements in water,” J. Acoust. Soc. Am., vol. 103, no. 5, pp. 23772384, 1998.

[64] J. G. Berryman, "Long-wavelength propagation in composite elastic media I. Spherical inclusions,” J. Acoust. Soc. Am., vol. 68, no. 6, pp. 1808-1819, 1980.

[65] J. G. Berryman, "Long-wavelength propagation in composite elastic media II. Elipsoidal inclusions,” J. Acoust. Soc. Am., vol. 68, no. 6, pp. 1820-1831, 1980.

[66] X. Yang and C. C. Church, “A model for dynamics of gas bubbles in soft tissue,” $J$. Acoust. Soc. Am., vol. 118, no. 6, pp. 3595-3606, 2005.

[67] A. Prosperetti, L. Crum and K. Commander, "Nonlinear bubble dynamics,” J. Acoust. Soc. Am., vol. 83, pp. 502-514, 1988.

[68] J. P. -Y. Maa and A. J. Mehta, “Soft mud properties: Voigt model,” Journal of Waterway, Port, Coastal, and Ocean Engineering, vol. 114, no. 6, pp. 765-770, 1988.

[69] H. Macpherson, "The attenuation of water waves over a non-rigid bed," J. Fluid Mech., vol. 97, no. 4, pp. 721-742, 1980.

[70] H.-T. Chou, M. A. Foda and J. R. Hunt, "Rheological response of cohesive sediments to oscillatory forcing,” in Nearshore and Estuarine Cohesive Sediment Transport, Coastal Estuarine Sci. Ser., vol. 42, Washington, D. C., AGU, 2013, pp. 126-147.

[71] M. A. Foda, J. R. Hunt and H.-T. Chou, "A nonlinear model for the fluidization of marine muds by waves,” Journal of Geophysical Research, vol. 98, no. C4, pp. 7039-7047, 1993.

[72] M. Jain and A. J. Mehta, "Role of basic rheological models in determination of wave attenuation over muddy seabeds,” Continental Shelf Research, vol. 29, pp. 642-651, 2009.

[73] K. W. Commander and A. Prosperetti, "Linear pressure waves in bubbly liquids: Comparison between theory and experiment,” J. Acoust. Soc. Am., vol. 85, pp. 732-746, 1989. 
[74] G. E. Claypool and I. R. Kaplan, "The origin and distribution of methane in marine sediments,” in Natural Gases in Marine Sediments, New York, Plenum Press, 1974, pp. 99-139.

[75] G. Mavko, T. Mukerij and J. Dvorkin, The Rock Physics Handbook, Cambridge University Press, 1998, pp. 1-511.

[76] R. Duraiswami, S. Prabhukumar and G. L. Chahine, "Bubble counting using an inverse acoustic scattering method,” J. Acoust. Soc. Am, vol. 104, pp. 2699-2717, 1998.

[77] T. G. Leighton, A. Mantouka, P. R. White and Z. Klusek, “Towards field measurements of populations of methane gas bubbles in marine sediment: An inversion method required for interpreting two-frequency insonification data from sediment containing gas bubbles," Hydroacoustics, vol. 11, pp. 203-224, 2008.

[78] A. Mantouka, Development of a two-frequency technique for gas-bubble sizing in marine sediment, PhD Thesis, University of Southampton, 2010.

[79] N. P. Chotiros and M. J. Isakson, “A broadband model of sandy ocean sediments: BiotStoll with contact squirt flow and shear drag," J. Acoust. Soc. Am. , vol. 116, no. 4, pp. 2011-2022, 2004.

[80] E. G. McLeroy and A. DeLoach, "Sound speed and attenuation, from 15 to $1500 \mathrm{kHz}$, measured in natural seafloor sediments,” J. Acous. Soc. Am., vol. 44, pp. 1148-1150, 1968.

[81] M. D. Richardson, K. B. Briggs, L. D. Bibee, P. A. Jumars, W. B. Sawyer, D. B. Albert, R. H. Bennett, T. K. Berger, M. J. Buckingham, N. P. Chotiros, P. H. Dahl, N. T. Dewitt, P. Fleischer, R. Flood, C. F. Greenlaw, D. V. Holliday and Hulbert, "Overview of SAX99: Environmental Considerations,” IEEE J. Ocean. Eng., vol. 26, pp. 26-53, 2001. 